\title{
A Novel Thermochemical Method for Fabrication and Theoretical Explanation of High Luminescent Mn-Doped CdS Nanoparticles
}

\author{
Mostafa Darvishi, Alireza Nikfarjam* \\ MEMS \& NEMS Laboratory, Faculty of New Sciences \& Technologies, University of Tehran, Tehran, Iran \\ Email: *a.nikfarjam@ut.ac.ir
}

How to cite this paper: Darvishi, M. and Nikfarjam, A. (2018) A Novel Thermochemical Method for Fabrication and Theoretical Explanation of High Luminescent Mn-Doped CdS Nanoparticles. Journal of Materials Science and Chemical Engineering, 6, 1-20.

https://doi.org/10.4236/msce.2018.63001

Received: November 29, 2017

Accepted: March 16, 2018

Published: March 19, 2018

Copyright $\odot 2018$ by authors and Scientific Research Publishing Inc. This work is licensed under the Creative Commons Attribution International License (CC BY 4.0).

http://creativecommons.org/licenses/by/4.0/

(c) (i) Open Access

\begin{abstract}
In this paper, fabrication and characterization of bare and doped CdS nanoparticles as well as investigating the luminescence properties of these particles as an important II-VI semiconductor are presented. A novel Thermochemical method was used for synthesis of these quantum dots. Thiols were used as the capping agent to prevent further growth during fabrication process. The application of TGA as a capping agent instead of TG was studied as a novel idea in this paper and was used practically in the synthesis of semiconductor nanoparticles. Using this process resulted in particles with sizes between $3-7$ $\mathrm{nm}$. Several samples were synthesized and characterized under various $\mathrm{Mn}$ ions doping ratio from 1:10 to $1: 180$, different temperatures from $40^{\circ} \mathrm{C}$ to $96^{\circ} \mathrm{C}$ and different $\mathrm{pH}$ values from 6 to 10 . Synthesis of CdS nanoparticles with high $\mathrm{Mn}$ ions concentration resulted in luminescence decrement, while luminescence of nanoparticles was increased by decreasing $\mathrm{Mn} / \mathrm{Cd}$ doping ratio until $\mathrm{Mn}: \mathrm{Cd}=1: 180$. The best fabrication temperature was obtained at $96^{\circ} \mathrm{C}$ and the highest luminescence was observed at the $\mathrm{pH}$ value of 9. A theoretical explanation for the behavior of fabricated high luminescent quantum dots is presented based on the principles of quantum mechanics.
\end{abstract}

\section{Keywords}

Mn:CdS Nanoparticle, Quantom Dot, Thermochemical Growth, Photoluminescence, UV-Vis Spectroscopy

\section{Introduction}

The optoelectronic properties of semiconductor nanoparticles are strongly dependent on their size, due to the well-known quantum confinement effects [1]. 
Among semiconductor nano-materials, CdS nanoparticles have attracted considerable attention due to their unique properties, which do not present in their bulk materials [2]. These unique properties introduce them as the important and useful materials for various applications such as solar cells, photodetectors, light-emitting diodes, photoelectrolysis and biological labels [3] [4] [5] [6].

Nanoparticles have been grown in a variety of ways, namely precipitation in the solid phase [7] [8], using porous materials as templates [9], growth in nano-sized micelles and precipitation in the liquid phase [10] [11].

In this paper, a thermochemical method to fabricate the bare and Mn-doped CdS nanoparticles of small sizes is developed. The nanoparticles are synthesized by reaction of sodium thiosulfate $\mathrm{Na}_{2} \mathrm{~S}_{2} \mathrm{O}_{3}$, cadmium sulfate $3 \mathrm{CdSO}_{4} \cdot 8 \mathrm{H}_{2} \mathrm{O}$ as sulfur and cadmium sources, respectively. Manganese nitrate $\left(\mathrm{Mn}\left(\mathrm{NO}_{3}\right)_{2} \cdot 4 \mathrm{H}_{2} \mathrm{O}\right)$ as $\mathrm{Mn}$ doping precursor and thioglycolic acid (TGA) $\mathrm{C}_{2} \mathrm{H}_{4} \mathrm{O}_{2} \mathrm{~S}$ as capping agent were also used. One of contributions of this paper is the utilization of TGA to improve the capping of nanoparticles and reach better optical properties. The as-prepared particles were analyzed by X-ray diffraction (XRD), transmission electron microscope (TEM), UV-VIS spectrometer, Fourier transform Infra-red (FTIR) and dynamic light scattering (DLS). Besides, $\mathrm{Mn} / \mathrm{Cd}$ doping ratio, temperature and $\mathrm{pH}$ value in fabrication processes were optimized to reach the highest possible luminescence.

This paper is structured as follow. A background of the previous works is presented in Section 2 emphasizing the state of art in the synthesis and characterization of CdS quantum dots. Section 3 draws the experimental procedures and results obtained under different conditions. The theoretical explanation of high luminescent CdS nanoparticles are discussed in Section 4 and finally the conclusion is drawn in Section 5.

\section{Background of the Previous Works}

Growth and fabrication of CdS nanoparticles was performed with some researchers [6] [12] [14] previously. The focus of each work was on the improvement of one parameter in the fabricated nanoparticles, while other criteria were not investigated carefully [1] [3] [15] [16]. One of the major contributions of this paper is fabrication and characterization of high luminescent Bare and $\mathrm{Mn}$-doped CdS nanoparticles while a sort of parameters and conditions are taken into account. This tendency toward the fabrication of high luminescent CdS nanoparticles provides an insight how the different environmental criteria could affect the synthesis process. The main objective in this paper is to investigate how the photoluminescence intensity and absorption spectra could vary in an acceptable range while different process conditions are applied.

The application of high luminescent bare and Mn-doped CdS nanoparticles is widely in optical devices such as optical limiting devices, display devices, optical switching and computing, phase conjugators and also optical correlators [17] [18] [19]. The influence of interfaces and intra-band transitions on the band gap 
of $\mathrm{CdS} / \mathrm{HgS}$ and GaN/X core-shell quantum dots were investigated in [20]. The core-shell model along with the distribution of radical wave functions was used for those quantum dots. A theoretical model to calculate the $1 \mathrm{~s}-1 \mathrm{~s}$ transition energy of an exciton in spherically layered quantum dots was presented in [20]. The main drawback in [20] is that the different environmental conditions were not taken into account in the presented model and the calculated functions. The defects related emission and nanosecond optical power limiting in CuS quantum dots was recently investigated in [21]. The nonlinear and optical properties of $\mathrm{CuS}$ quantum dots were reported through a one-pot synthesis technique. The effect of surface states in the luminescent quality of those quantum dots was presented while no evidence for the behavior of surface states under different environmental conditions was provided which can be addressed as one of the drawbacks in [21]. A co-precipitation method with microwave assistant was employed for fabrication of $\mathrm{ZnO}$ nanorods in [22]. Those nanorods were introduced as the recyclable photocatalysts. Temperature variation is one of the major affecting parameters in the growth of zinc salt nanoparticles which was not considered in details in [22]. Synthesis and characterization of differently structured $\mathrm{CdS} / \mathrm{ZnS}$ sandwich and core-shell nanocomposites was recently investigated in [23]. A simple and modified chemical precipitation method under ambient conditions was used for the fabrication of those nanocomposites. The optical properties for $\mathrm{CdS} / \mathrm{ZnS}$ nanocomposites were investigated but due to a lack of precise control on the ambient conditions, the results did not show a good agreement than those expected from the behavior of sandwich and core-shell nanocomposites.

\section{Experimental}

In this section the experiments for fabricating high luminescent CdS nanoparticles are presented. The CdS nanoparticles were grown by a chemical precipitation method at different doping concentrations, different temperatures and different $\mathrm{pH}$ values. The sodium thiosulfate and extra pure cadmium sulfate were used as the reactant materials and TGA was added as the capping agent. The concentrations of $\mathrm{CdSO}_{4}$ and $\mathrm{Na}_{2} \mathrm{~S}_{2} \mathrm{O}_{3}$ were $5 \mathrm{mM}$ and $50 \mathrm{mM}$, respectively. The concentration of TGA was $0.01 \mathrm{M}$. Mn doping concentration to cadmium concentration was variable in the range of $\mathrm{Mn}: \mathrm{Cd}=1: 10$ to $\mathrm{Mn}: \mathrm{Cd}=1: 180$. In a typical procedure, a $30 \mathrm{ml}$ of $\mathrm{Na}_{2} \mathrm{~S}_{2} \mathrm{O}_{3}$ solution was prepared and TGA drop-wise was added into the solution. Then, $\mathrm{CdSO}_{4}$ was added with pressure to make an agitation in the solution. Sounds that adding the cadmium thiosulfate with pressure into the solution results in a great effect on the size distribution of CdS nanoparticles. The $\mathrm{pH}$ value was adjusted by adding the appropriate amount of $\mathrm{NH}_{4} \mathrm{OH}$ while adding TGA. The $\mathrm{pH}$ was tuned on a desired value for process optimization. The reaction took place in a bath with a controlled temperature. The temperature was tuned from room temperature to $96^{\circ} \mathrm{C}$ for different fabrication processes under different $\mathrm{Mn}$ doping concentrations. In all experiments, 
the samples were cleaned with a standard procedure in ultrasonic bath.

UV-Vis absorption spectroscopy method was employed to monitor the growth of nanoparticles and measure the luminescence properties. An Ocean Optics HR4000 fiber-based spectrometer was employed for the UV-Vis spectroscopy. A UV-Violet laser diode at about $400 \mathrm{~nm}$ wavelength was used for the excitation of fabricated nanoparticles. For X-ray diffraction (XRD) measurements, the nanoparticles were extracted by centrifuging and spinned on a glass substrate. The measurements were carried out using a Bruker D4 X-ray diffractometer. The $\mathrm{Cu} \mathrm{Ka}(1.54 \AA)$ X-ray line was used as the probe beam. Also TEM was done using $100 \mathrm{kV}$ PHILIPS EM2085 microscope. Besides, Fourier Transform Infrared Spectroscopy (FTIR) Analysis performed to obtain material bonds.

Following the experiments, the approximate size of nanoparticles was estimated and compared with DLS analysis results.

Following this section, different scenarios for fabrication of high luminescent CdS nanoparticles under different conditions are presented. These scenarios contain fabrication of bare and $\mathrm{Mn}$-doped CdS nanoparticles with different $\mathrm{Mn}$ dpoing concentrations, different temperatures and different $\mathrm{pH}$ values.

\subsection{Fabrication of CdS Nanoparticles with Different Manganese Doping Concentrations}

The materials used in this section are the same as those mentioned earlier at the beginning of Section III. In this case, the temperature was adjusted to $96^{\circ} \mathrm{C}$, the heating time was 60 minutes and the $\mathrm{pH}$ value was kept at 8 using $\mathrm{NH}_{4} \mathrm{OH}$ solution. The main key point of experiment is to keep $\mathrm{pH}$ and temperature constant at their tuned values. Figure 1 shows the absorption spectra for CdS:Mn nanoparticles grown with a thermochemical method. Obviously, the sample with doping level of $\mathrm{Mn}: \mathrm{Cd}=1: 20$ has the highest absorption compared to the other samples. Also, the absorption spectra of these samples confirm that the largest bandgap energy belongs to the sample with doping level of $\mathrm{Mn}: \mathrm{Cd}=1: 20$.

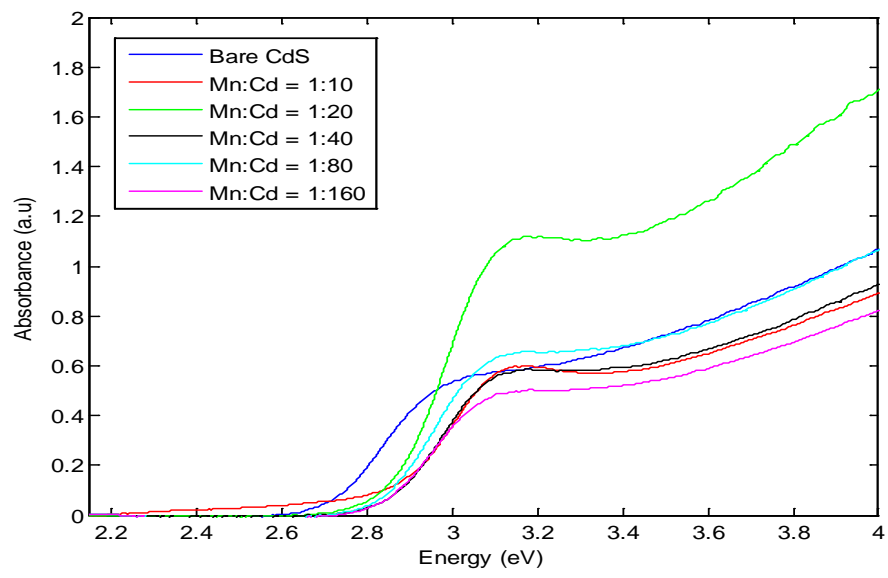

Figure 1. Absorption spectra of CdS:Mn nanoparticles with different doping ratios versus energy. 
In order to investigate the photoluminescence properties of fabricated CdS:Mn nanoparticles with a thermochemical method, a $400 \mathrm{~nm}$ UV laser diode was used as an excitation source. The photoluminescence (PL) spectra of samples as a function of wavelength are shown in Figure 2. The peaks position in each sample is considered to explain the dynamics of photoluminescence.

In the synthesized bare CdS nanoparticles, there is a peak near $605 \mathrm{~nm}$ that is due to the recombination of a trapped electron in sulfur vacancies with a hole in CdS valence band. In this process, inadequate concentration of sulfur maybe results in sulfur vacancies. In this case, a photo excited electron will be trapped in sulfur vacancies and will produce a red shift in PL spectrum. While exciting the CdS nanoparticles with UV laser, the electrons of valence band will be excited into the conduction band and will remain holes in the valence band.

In Figure 3, the sample with doping of $\mathrm{Mn}: \mathrm{Cd}=1: 20$ has more PL intensity than the sample with doping level of $\mathrm{Mn}: \mathrm{Cd}=1: 10$ and introduces a peak near $495 \mathrm{~nm}$ which produces green emission due to sulfur intermediates.

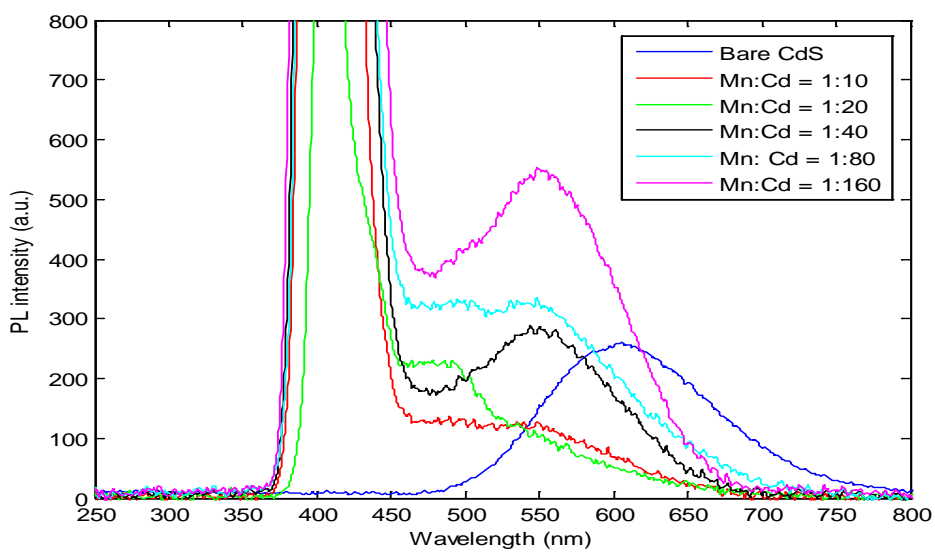

Figure 2. PL intensity of samples prepared in thermochemical method.

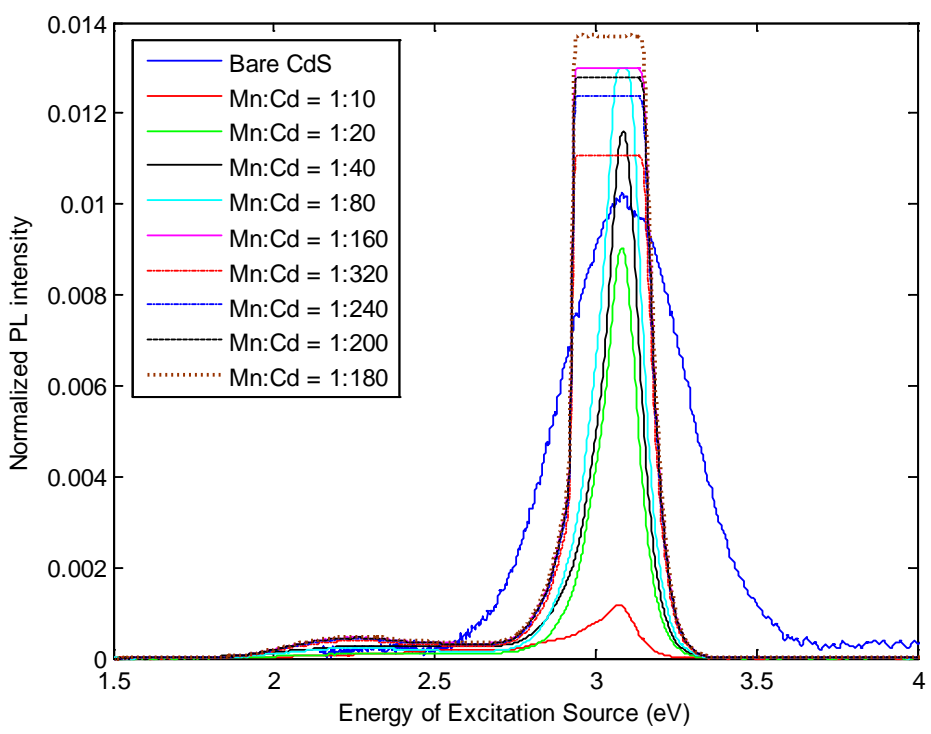

Figure 3. PL intensity of samples prepared in thermochemical method. 
The sample with doping level of $\mathrm{Mn}: \mathrm{Cd}=1: 40$ has a peak in $548 \mathrm{~nm}$ that results in yellow emission caused by cadmium intermediates $\left(I_{C d}^{+}\right)$. The sample with doping $\mathrm{Mn}: \mathrm{Cd}=1: 80$ has two peaks near $500 \mathrm{~nm}$ and $550 \mathrm{~nm}$ that are due to the sulfur vacancies and $\mathrm{Mn}^{2+}$ ions (orange emission), respectively. The sample with doping $\mathrm{Mn}: \mathrm{Cd}=1: 160$ has a peak near $548 \mathrm{~nm}$ (yellow emission) due to the cadmium intermediates $\left(I_{C d}^{+}\right)$. In order to figure out the best PL intensity of Mn doped CdS nanoparticles, a set of CdS:Mn nanoparticles with doping levels of $\mathrm{Mn}: \mathrm{Cd}=1: 180,200,240$ and 320 were produced and their PL intensity was measured. Figure 3 illustrates the photoluminescent intensity of prepared samples with different doping levels. It is obvious when the doping level is tuned to $\mathrm{Mn}: \mathrm{Cd}=1: 180$, the highest photoluminescence is achieved.

FTIR analysis was performed in order to find out the bonds of Mn doped CdS nanoparticles Figure 4 shows the FTIR analysis result for fabricated nanoparticles with doping $\mathrm{Mn}: \mathrm{Cd}=1: 180$ including all possible bonds.

The Transmission Electron Microscopy (TEM) image of this sample shown in Figure 5 that confirms the spherical structure for Mn doped CdS nanoparticles. The size distribution regime was investigated through DLS technique shown in Figure 6 . The results show a $3-7 \mathrm{~nm}$ size distribution with a peak of $5 \mathrm{~nm}$ for doped nanoparticles.

The crystalline properties of Mn-doped CdS semiconductor nanoparticles fabricated at $96^{\circ} \mathrm{C}$ with doping level of $\mathrm{Mn}: \mathrm{Cd}=1: 180$ at $\mathrm{pH}=9$ was investigated via $\mathrm{XRD}$ analysis as shown in Figure 7. According to the $\mathrm{XRD}$ result, the dominant crystalline structure of the sample is hexagonal. This analysis was taken

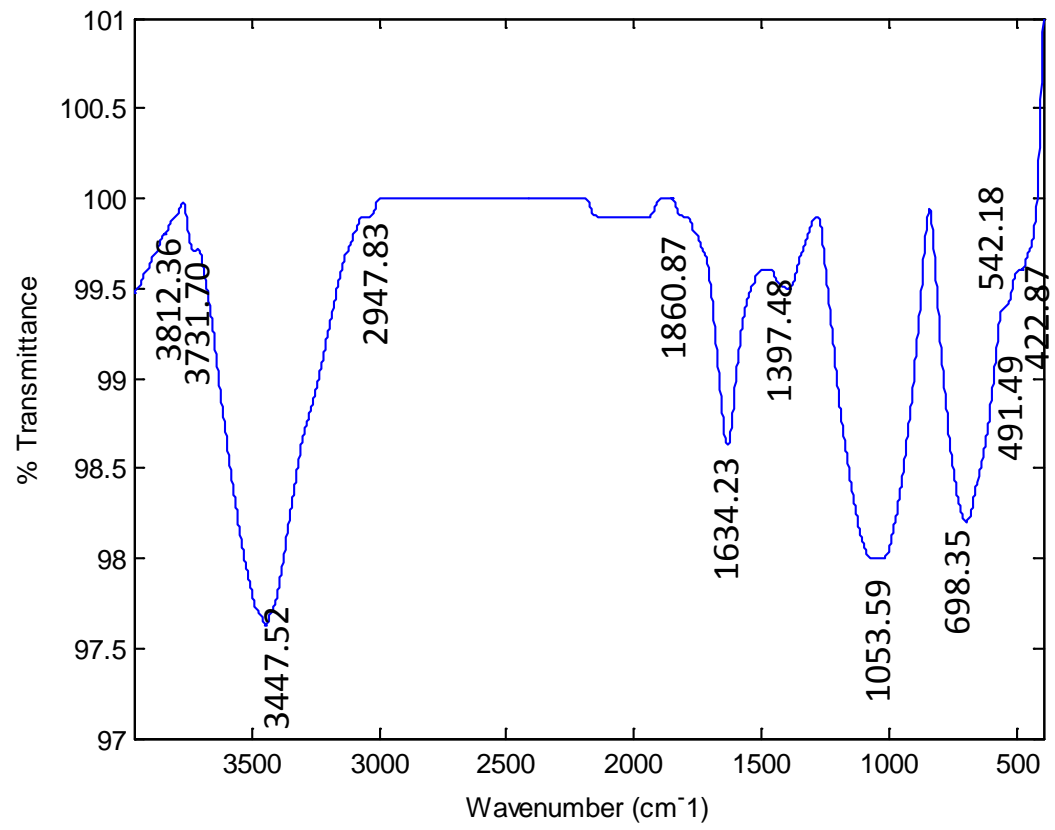

Figure 4. FTIR result of sample prepared in thermochemical method. $\{422.87$, 491.49: CdS Stretching vibration|542.18: Mn to CdS bond|698.35: Thiol bond due to TGA existence|1053.59: first-type alcohol C-O|1397.48: S-O bond|1634.23, 3447.52, 3731.70, 3812.36: O-H bond|1860.87: Mn-S bond|2947.83: C-H bond\}. 


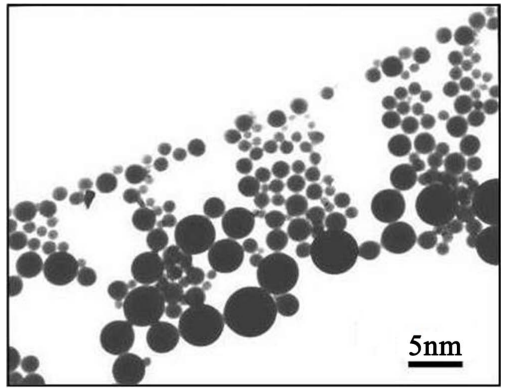

Figure 5. TEM image of sample with doping $\mathrm{Mn}: \mathrm{Cd}=1: 180$ fabricated using thermochemical method.
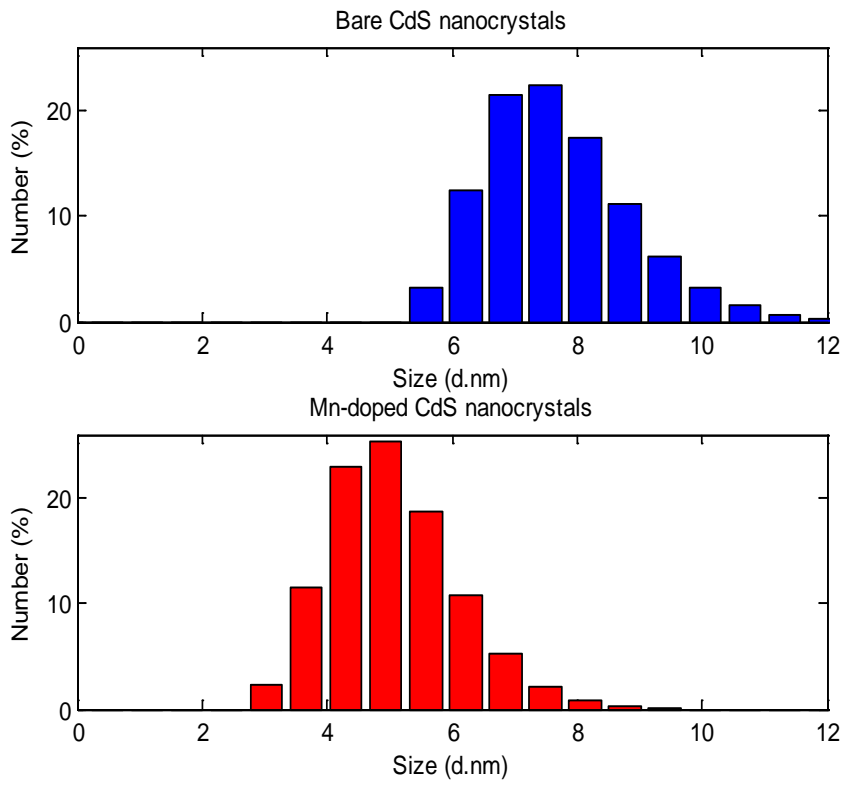

Figure 6. Size distribution of bare and Mn-doped CdS nanoparticles doping $\mathrm{Mn}: \mathrm{Cd}=1: 180(\mathrm{pH}=8)$.

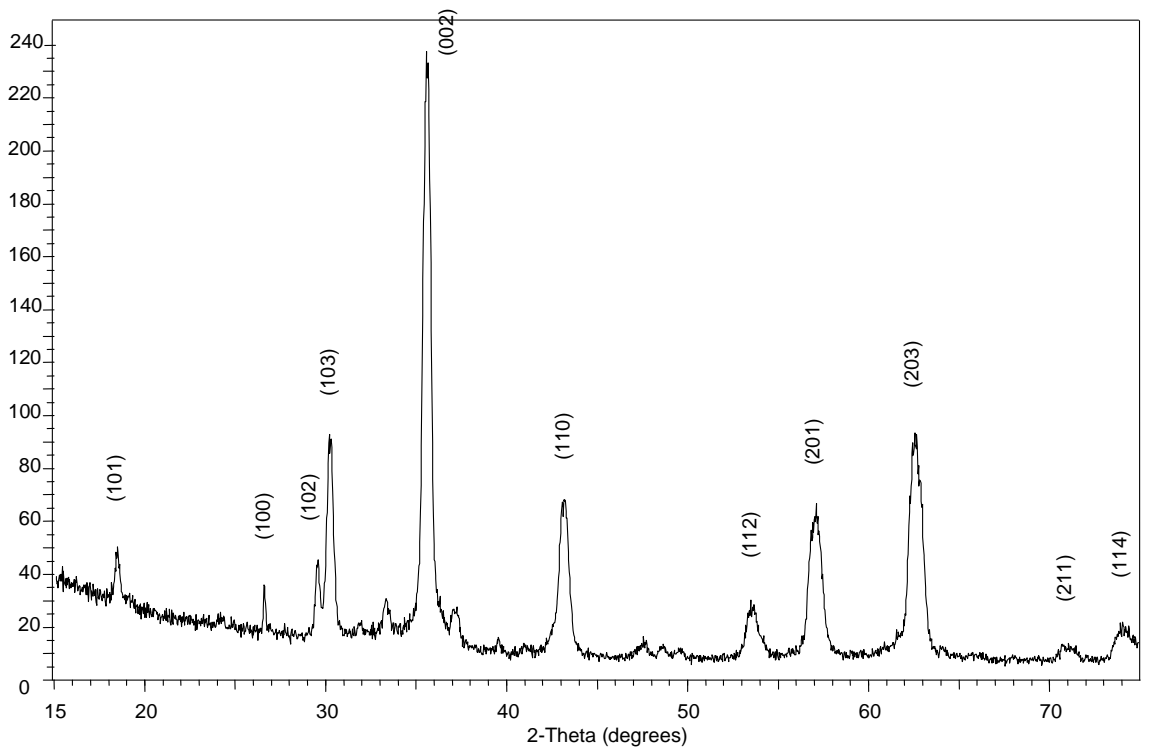

Figure 7. Characterization of fabricated crystalline nanoparticles via XRD analysis. 
with $\mathrm{a}=3.8200 \AA \AA$ and $\lambda_{\mathrm{X} \text {-Ray }}=1.54 \AA$, respectively. The Scherer's formula depicted in (15) is used to calculate the size of nanoparticles where $\mathrm{D}$ is the crystalline size, $\lambda$ is X-ray wavelength, $\beta$ is full width at half maximum (FWHM) and $\theta$ is the diffraction angle.

$$
D=\frac{0.9 \lambda}{\beta \cos \theta}
$$

\subsection{Fabrication of Mn-Doped CdS Nanoparticles at Different Temperatures}

In this section, the Mn-doped CdS nanoparticles were fabricated at different temperatures in order to understand the effect of temperature on the size distribution and photoluminescence properties of doped nanoparticles. The doped quantum dots were fabricated with doping level of $\mathrm{Mn}: \mathrm{Cd}=1: 180$ which achieved the best PL intensity in the former section. The temperature of nanoparticle growth was changed between $40^{\circ} \mathrm{C}$ and $96^{\circ} \mathrm{C}$. Figure 8 shows the absorption spectra of Mn-doped CdS nanoparticles at different growth temperatures. Obviously, the absorption edge has a blue shift with temperature decrement that confirms the smaller size distribution at low temperatures.

The PL spectra of Mn-doped CdS nanoparticles at different growth temperatures is shown in Figure 9. The sample prepared at $96^{\circ} \mathrm{C}$ has a peak around 550 $\mathrm{nm}$ that refers to orange emission caused by $\mathrm{Mn}^{2+}$ ions' effect. The PL intensity of this sample is higher than the others. The sample prepared at $70^{\circ} \mathrm{C}$ has a peak around $500 \mathrm{~nm}$ that belongs to the sulfur intermediates, while no effect of $\mathrm{Mn}^{2+}$ luminescence was observed in this spectrum.

As shown in Figure 9, the PL intensity of sample prepared at $70^{\circ} \mathrm{C}$ was decreased due to the shallow traps caused by $\mathrm{Mn}^{2+}$ ions just below the conduction band. While temperature decrement, these shallow traps could not be removed by heating and will trap the electrons of conduction band and produce the orange emission. Therefore, the PL quality caused by CdS nanoparticles will be decreased despite the small size distribution of nanoparticles. As shown in Figure 9, the sample prepared at $70^{\circ} \mathrm{C}$ has lower PL intensity with a peak around $475 \mathrm{~nm}$ and a blue shift that confirms lowering the size distribution in fabricated nanoparticles. Again, the decrement of PL intensity in this sample is caused by shallow traps under the conduction band. The last sample prepared at $40^{\circ} \mathrm{C}$ has the least PL intensity due to the dominant photoluminescence of $\mathrm{Mn}^{2+}$ ions rather than CdS nanoparticles.

\subsection{Fabrication of Mn-Doped CdS Nanoparticles with Different pH Values}

The objective of this section is to investigate the effect of $\mathrm{pH}$ value of growth media on the size distribution and photoluminescence quality of fabricated Mn-doped CdS quantum dots. Mn-doped CdS semiconductor nanoparticles with doping level of $\mathrm{Mn}: \mathrm{Cd}=1: 180$ were fabricated at different $\mathrm{pH}$ values. Four 
samples including former fabrication conditions with $\mathrm{pH}$ values of $6,8,9$ and 10 were prepared.

Figure 10 shows the PL spectra of fabricated nanoparticles with doping level of $\mathrm{Mn}: \mathrm{Cd}=1: 180$ at different $\mathrm{pH}$ values. The sample prepared at $\mathrm{pH}=6$ has the

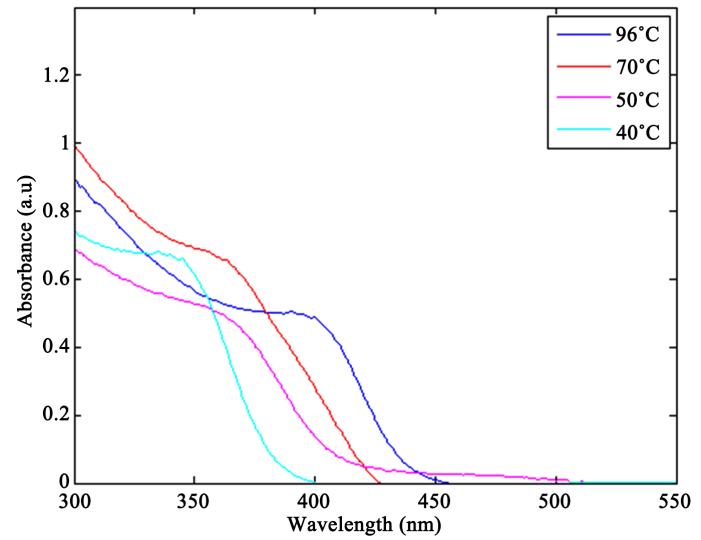

Figure 8. Absorption spectra of CdS:Mn nanoparticles at different temperatures.

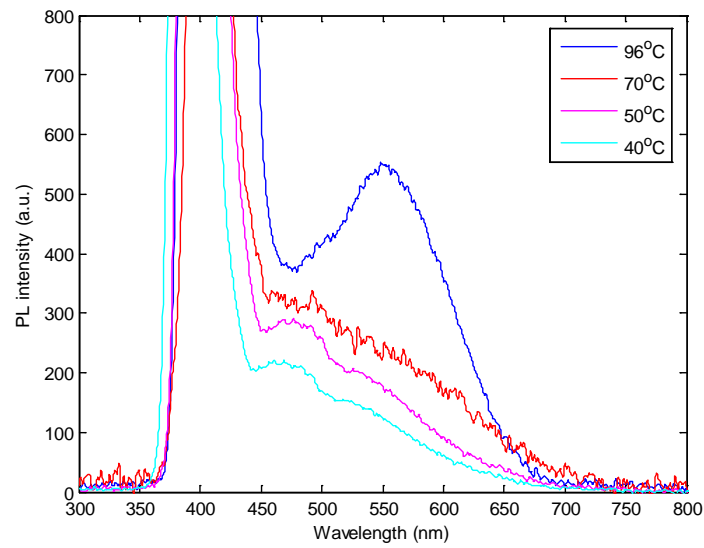

Figure 9. PL intensity spectra of fabricated nanoparticles at different temperatures.

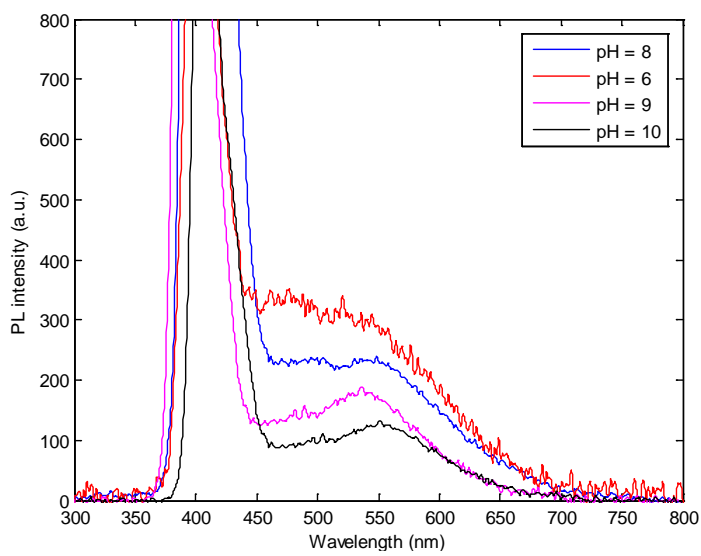

Figure 10. PL intensity of fabricated nanoparticles at different $\mathrm{pH}$ values. 
highest photoluminescence intensity that confirms the least size distribution for nanoparticles with two peaks around $495 \mathrm{~nm}$ and $535 \mathrm{~nm}$ that are referred to sulfur intermediates and orange emission due to $\mathrm{Mn}^{2+}$ ions, respectively. The sample fabricated at $\mathrm{pH}=8$ has less photoluminescence intensity due to spread size distribution of nanoparticles and has two peaks near $500 \mathrm{~nm}$ and $550 \mathrm{~nm}$. The sample prepared at $\mathrm{pH}=9$ has less photoluminescence intensity compared to two former samples with a peak around $540 \mathrm{~nm}$ that is referred to orange emission caused by $\mathrm{Mn}^{2+}$ ions. This spectrum does not have any peak around $500 \mathrm{~nm}$. This phenomenon is due to the compensation of sulfur intermediates with $\mathrm{pH}$ increase. In this case, at higher $\mathrm{pH}$ values the density of $\mathrm{H}^{+}$ions is decreased, so the effective radius will be decreased. The reaction occurs around the surface of nanoparticles and will increase the size distribution of particles. This phenomenon will produce some sulfur intermediates. The sample with $\mathrm{pH}=10$ has the least photoluminescence intensity that confirms the wider size distribution of nanoparticles.

\section{Discussion}

In this section, the theoretical aspect behind the fabrication of high luminescent CdS nanoparticles under different conditions is presented in order to justify the results obtained by experiments.

\subsection{Solving the Symmetric Potential Problem for A CdS Semiconductor Nanoparticles}

Since a large number of nanocrystals have a spherical structure (according to TEM image), so it is possible to consider the symmetric potential for this materials [24]. The well-known Hamilton's Equation with symmetric radius is as follow:

$$
H=-\frac{\hbar^{2}}{2 m} \nabla^{2}+U(r)
$$

where, $m$ is the mass of nanoparticle and $U(r)$ indicates the radical potential. So, the Laplace equation [25] [26] in spherical coordination is as Equation (2):

$$
\nabla^{2}=-\frac{\partial}{r^{2} \partial r}\left(r^{2} \frac{\partial}{\partial r}\right)-\frac{1}{r^{2} \sin v}\left[\frac{\partial}{\partial v}\left(\sin v \frac{\partial}{\partial v}\right)+\frac{\partial^{2}}{\sin v \partial \varphi^{2}}\right]
$$

The solution for Equation (2) is as shown in Equation (3).

$$
\Psi_{n, l, m}(r, v, \phi)=\frac{u_{n, l}(r)}{r} Y_{l m}(v, \phi)
$$

In Equation (3), the wave function $\Psi_{n, l, m}$ is divided into two terms: the radical mode with spherical symmetric wave function $u_{n, l}$, and Laguerre polynomials $Y_{l m}(v, \phi)$ [27] [28] [29]. The eigenvalues of $E_{n, l}$ in the radical part of Hamilton's equation depends just on the spherical potential.

$$
\left[-\frac{\hbar^{2}}{2 m r^{2}} \frac{d^{2}}{d r^{2}}+U(r)+\frac{\hbar^{2}}{2 m} l(l+1)\right] u_{n, l}(r)=E_{n, l} u_{n, l}(r)
$$


where, $U(r)$ is the spherical potential. The solutions of Equation (4) are achieved with three quantum numbers: the fundamental quantum number $n$, the quantum number of angular momentum $l$, and the particle's mass $m$ which its magnitude is in $\mathrm{z}$-axis direction. The potential function $U(r)$ is considered as follow:

$$
U(r)= \begin{cases}0 & r \leq a \\ \infty & r>a\end{cases}
$$

Also, the eigenvalues of energy are obtained from spherical Bessel functions $X_{n l}$.

$$
E_{n, l}=\frac{\hbar^{2} X_{n l}^{2}}{2 m a^{2}}
$$

The first value of spherical Bessel function $\left(X_{10}\right)$ equals to $\pi$. Since this function has an inverse square relation with radius, so, the large quantization energy will be obtained for the small nanocrystals. Different regimes of confinement for nanocrystals as the weak and strong confinement are considered by solving Equation (6).

According to the calculated values of $X_{n l}$ in Table 1, the eigenvalues for a CdS semiconductor particle with mass of $m$ are obtained. Based on the experimental results obtained by DLS technique, $80 \%$ amount of nanoparticles have the size of $5 \mathrm{~nm}$. So, the eigenvalues of energy are calculated with approximation of a $=5 \mathrm{~nm}$. The calculation results for $\hbar^{2} X_{n l}^{2} / 2 a^{2}$ are shown in Table 2.

Table 1. Values of spherical bessel functions.

\begin{tabular}{lccc}
\hline 1 & $\mathrm{n}=1$ & $\mathrm{n}=2$ & $\mathrm{n}=3$ \\
\hline 0 & 3.14 & 6.28 & 9.42 \\
1 & 4.49 & 7.73 & -- \\
2 & 5.72 & 9.10 & -- \\
3 & 6.99 & 10.42 & -- \\
4 & 8.18 & -- & -- \\
5 & 9.32 & -- & - \\
\hline
\end{tabular}

Table 2. Calculated eigenvalues of energy.

\begin{tabular}{cccc}
\hline$I$ & $\mathrm{n}=1$ & $\mathrm{n}=2$ & $\mathrm{n}=3$ \\
\hline 0 & $8.576 \mathrm{E}-14$ & $3.431 \mathrm{E}-13$ & $7.719 \mathrm{E}-13$ \\
1 & $1.753 \mathrm{E}-13$ & $5.198 \mathrm{E}-13$ & - \\
2 & $2.846 \mathrm{E}-13$ & $7.203 \mathrm{E}-13$ & -- \\
3 & $4.250 \mathrm{E}-13$ & $9.445 \mathrm{E}-13$ & -- \\
4 & $5.820 \mathrm{E}-13$ & -- & -- \\
5 & $7.556 \mathrm{E}-13$ & -- & -- \\
\hline
\end{tabular}




\subsection{Average Radius and Bandgap Calculation for CdS Semiconductor Nanoparticles}

The bandgap of CdS semiconductor nanoparticles can be assumed as the following:

$$
\alpha=A\left(h v-E_{g}\right)^{n} / h v
$$

where, $\alpha$ is the absorption coefficient, $E_{g}$ is the absorption bandgap, $A$ is a scalar constant, and $n$ depends on transition type and could be considered as $1 / 2,2,3 / 2$ or 3 that corresponds to direct allowed transitions, indirect allowed transitions, direct forbidden transitions and indirect forbidden transitions, respectively. Since the CdS semiconductor nanoparticles have direct allowed transitions, so $n=1 / 2$ in Equation (7).

In this section, a new equation is provided in order to estimate the average radius for CdS semiconductor nanoparticles. This equation is based on absorption spectrum characteristics as shown in Equation (8).

$$
R=h\left(8 \mu \Delta E_{g}\right)^{-1 / 2}
$$

where, $h$ is Plank's constant, $\mu=\left(m_{e}^{*}\right)^{-1}+\left(m_{h}^{*}\right)^{-1}$ which $m_{e}^{*}=0.2 m_{e}$ and $m_{h}^{*}=0.81 m_{e}$ for CdS nanoparticles, and $\Delta E_{g}$ is the difference between bandgap energy of nanoparticles $E_{g}^{0}$ and the bulk semiconductor $E_{g}$. For CdS nanoparticles, $E_{g}=2.4 \mathrm{eV}$ and $E_{g}^{0}$ are obtained from absorption spectrum as shown in Equation (9).

$$
E_{g}^{0}(\mathrm{eV})=12397.8 / \lambda(\AA)
$$

\subsection{Arrangement of Doping Atoms in CdS Nanocrystals}

In photoluminescence phenomenon, the solid crystal is exited with photonic absorption. The absorption and transmission wavelengths are different and the transmission energy is smaller than the absorption energy [30]. The frequency transmission is also called the Stocks transmission [31]. The ionic doping such as $\mathrm{Mn}^{2+}$ can surround the surface of $\mathrm{CdS}$ nanocrystals. Effect High concentration of capping agent results in growth of nanoparticles with small size regime due to the capping of nanoparticles' surface with capping agent [12]. On the other hand, decreasing the amount of agent material will increase the surface traps that are visible in photoluminescence spectrum. The UV-Vis spectrum analysis in experiments showed that the spreading of emission band is due to the wider size distribution of nanoparticles [15] [32]. The Thioglycolic acid (TGA) or $\mathrm{C}_{2} \mathrm{H}_{2} \mathrm{O}_{2} \mathrm{~S}$ was used as the capping agent in our experiments. The structure of the TGA molecule is shown in Figure 11.

The energy band diagram for CdS nanoparticles was utilized in order to understand the effect of TGA on the nanoparticles' growing process [33]. As shown in Figure 12, the cadmium surface ions produce some states in the bandgap just below the conduction band also called electron traps, and the sulfur surface ions produce some states in the bandgap just above the valance band called hole 


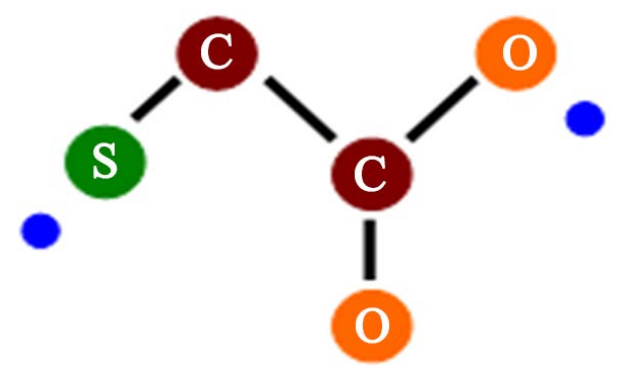

Figure 11. Structure of Thioglycolic acid.

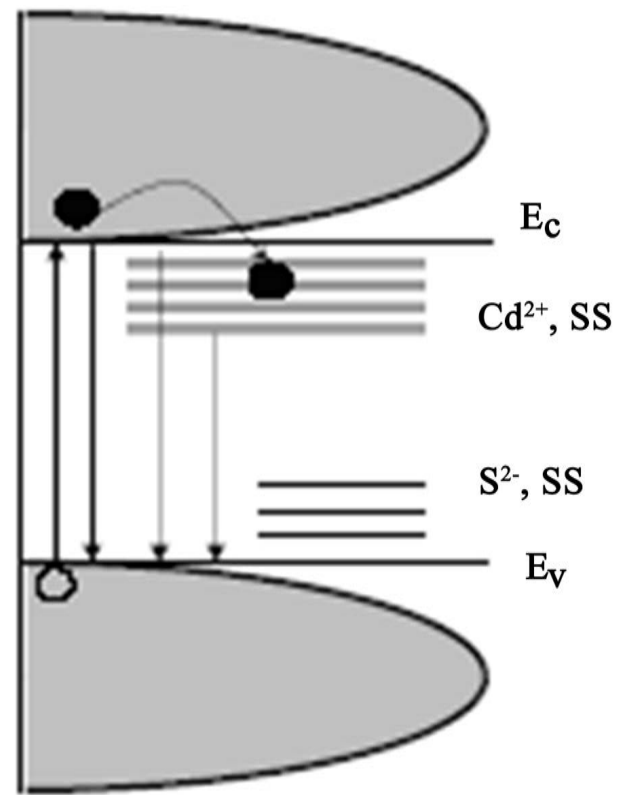

Figure 12. Energy band diagram of CdS nanoparticles.

traps. If the surface is free from absorbed impurities, the photo excited electrons of conduction band will be trapped in $\mathrm{Cd}^{2+}$ states and the holes of valence band will be trapped in $\mathrm{S}^{2-}$ states. In the solution phase, sulfur atoms are saturated with hydroxyl bonds and hence, the photoluminescence of nanoparticles will be dominated due to the recombination of electrons in conduction band and also the recombination of $\mathrm{Cd}^{2+}$ surface states with holes in the valence band.

Most of the molecules join together after a few moments. this removes the surface states of $\mathrm{Cd}^{2+}$ ions which decreases the surface states-to-band emission and increases band-to-band emission [3].

Our results showed wide wavelength emission near $500-600 \mathrm{~nm}$ which is due to the surface states created by sulfur vacancies. Using an organic agent material like TGA, capping of $\mathrm{Cd}^{2+}$ ions instead of $\mathrm{S}^{2-}$ ions results in a wide wavelength emission.

\subsection{Luminescence Mechanism in Bare CdS and Mn-Doped CdS Nanoparticles}

The luminescence mechanism in the bare and Mn-doped CdS semiconductor 
nanoparticles was studies in order to verify the luminescence quality of CdS and CdS:Mn nanoparticles [13]. The photoluminescence spectrum (PL) for CdS nanocrystals contains three emission bands, green, yellow and red. The green and yellow emissions address the band-edge emissions, while the red band belongs to the surface defects or the surface states of nanoparticles [34].

Also, it was found that when the CdS nanocrystals are not capped with capping molecules, the red band will be dominant and determines the PL spectrum for Nanocrystals. The band in the range of $700-800 \mathrm{~nm}$ addresses the complex defects including cadmium or sulfur vacancies.

In CdS nanoparticles, the cadmium vacancies and the sulfur intermediates act as acceptor, while the sulfur vacancies and cadmium intermediates will be the donors [14].

The energy diagram shown in Figure 13 represents the different transitions in CdS nanoparticles, where:

1) the band-to-band transmission.

2) the free exciton recombination.

3) the exciton mutation for donor neutralization.

4) the exciton mutation for acceptor neutralization.

5) the donor-acceptor pairs.

6) the excitation from sulfur intermediates $I_{S}^{-}$to the conduction band, results in green emission.

7) the yellow emission due to the cadmium intermediates $I_{C d}^{+}$.

8) the red emission due to the sulfur vacancies.

9) the excitation from cadmium vacancies to the valance band.

It was found that the bare CdS nanoparticles usually have lower PL intensity due to the created surface states in their unsaturated bands [3]. Also, when the bare CdS nanoparticles deal with free space, they won't be stable due to the photo-oxidation phenomenon. The main reason is the lack of sulfur vacancies that causes the photoexcited electrons in CdS. So, the weak PL emission is due to the lack of luminescence centers in nanoparticles' structure [35]. Also, it was found that increasing the ratio of $\mathrm{Cd}^{2+}$ ion in nanoparticles' structure results in increasing the sulfur vacancies and results in PL increment in bare CdS nanoparticles. Also, our studies show that the sulfur vacancies, $\mathrm{S}^{2-}$, act as deep traps for the photogenerated electrons in the conduction band and result in red emission in CdS nanoparticles. In this case, it is possible to have no band-edge luminescence due to the agglomeration of nanoparticles.

The four-state photoluminescence mechanism for Mn-doped CdS nanoparticles is shown in Figure 14. This mechanism is explained as follow: 1) The photoexcitation in inner energy levels of CdS nanoparticles occurs; 2) a large number of photoexcited carriers are trapped with the localized surface states; 3 ) the energy of these trapped carriers is transferred from surface states to $\mathrm{Mn}^{2+}$ ions via a recursive reaction; 4) the emission from centers of $\mathrm{Mn}^{2+}$ ions along with radiative recombination in the surface states [36]. 


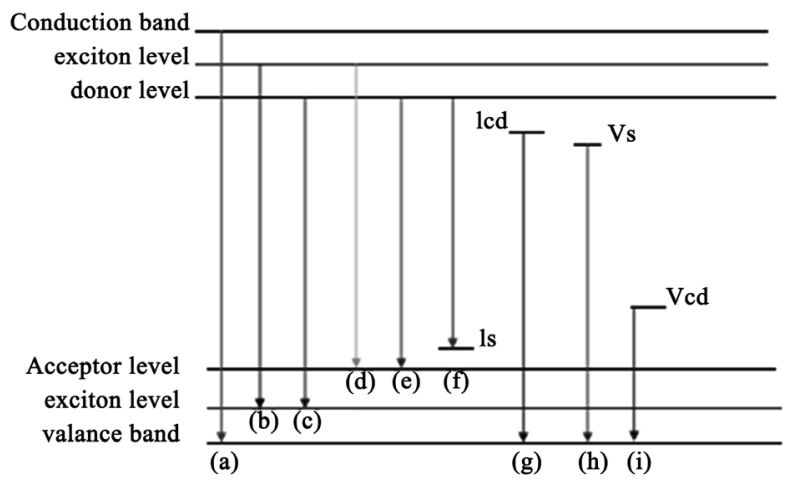

Figure 13. Energy levels diagram based on different transitions in PL spectrum.

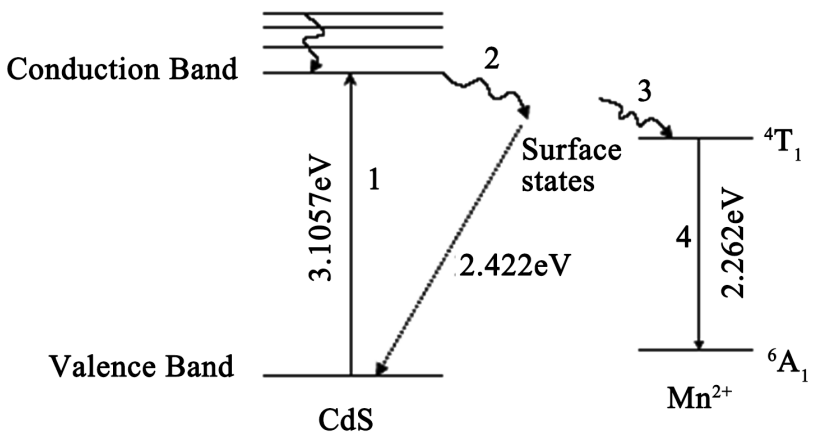

Figure 14. Photoluminescence mechanism in CdS:Mn nanoparticles. The numerical energy values are obtained from our experiments.

\subsection{Effects of Temperature, Mn Doping and $\mathrm{pH}$ Value on the Photoluminescence Mechanism of Mn-Doped CdS Nanoparticles}

Figure 14 also depicts the temperature dependency of the photoluminescence of Mn-doped CdS nanoparticles. After excitation of host CdS, 1) the excited charge carriers are trapped in shallow trap states. Suppose that the electrons are trapped in the states just below the conduction band 2). Trapping of charge carriers is followed by either energy transfer to the ${ }^{4} T_{1}$ excited state of a $\mathrm{Mn}^{2+}$ ion 3), or is followed by a radiative recombination with a deep trapped hole in a defect state 4).

Step 3 is followed by a radiative decay from ${ }^{4} T_{1}$ excited state to ${ }^{6} A_{1}$ ground state of $\mathrm{Mn}^{2+}$ ion 5) that results in orange emission due to the dominance of doping ions. The shallow traps just below the conduction band will be thermally removed while increasing the temperature results in decreasing of defect related CdS emission and also decrement of $\mathrm{Mn}^{2+}$ emission. If the excited state ${ }^{4} T_{1}$ of $\mathrm{Mn}^{2+}$ ions was directly filled with electrons of conduction band 6), so maybe the emission due to CdS and the emission due to $\mathrm{Mn}^{2+}$ ions have different quenching temperatures [2] [3] [7].

The PL spectrum confirms that the Mn-doped CdS nanocrystals contain a large number of $\mathrm{Mn}^{2+}$ ions that produce a non-uniform distribution of $\mathrm{Mn}^{2+}$ 
transition energy states in the nanocrystal structure [37]. The transition energy between 3d-level of $\mathrm{Mn}^{2+}$ ions is strongly depended to the field intensity around the $\mathrm{Mn}^{2+}$ ions [16]. The transition energy of ${ }^{4} T_{1} \rightarrow{ }^{6} A_{1}$ that is sensitive to the surrounded environment in $\mathrm{Mn}^{2+}$ ions produces a wide PL spectrum strongly depended to the excitation intensity [38]. This effect was investigated in experimental section with various $\mathrm{Mn}^{2+}$ doping concentrations.

The effect of $\mathrm{pH}$ on the photoluminescence intensity of $\mathrm{CdS}$ nanoparticles was also studied. Increasing $\mathrm{pH}$ value will cause a red shift in the absorption edge. To explain this phenomenon, we focused on the $\mathrm{H}^{+}$concentration in the chemical reaction [39].

It seems that there is a local source of $\mathrm{H}^{+}$ions near the surface of nanoparticles in the presence of TGA that speeds up the reaction process when $\mathrm{pH}>8$. This replaced source of $\mathrm{H}^{+}$ions can be the thiols' molecules that propagate $\mathrm{H}^{+}$ ions while sticking to $\mathrm{Cd}^{+}$ions. So, the $\mathrm{pH}$ is increased near the surface of nanoparticles and as a result increases the size distribution [40].

Since, all of the conditions are constant during the fabrication process; we can attribute the particle's mass to the process time. With the assumption of spherical shape for nanoparticles with diameter $d$, the mass $m$ is denoted as density, the number of nanoparticles $n$, and the volume of each particle. Using the EMA method, the following formula is obtained.

$$
m=\rho n \frac{\pi}{6} d^{3}=\rho n \frac{\pi}{6}\left[2 \pi^{2} \hbar^{2}\left(\frac{1}{m_{e}^{*}}+\frac{1}{m_{h}^{*}}\right) \frac{1}{\Delta E_{g}}\right]^{\frac{3}{2}} \propto t
$$

where, $m_{e}^{*}$ and $m_{h}^{*}$ are respectively the electron and hole's effective mass in CdS nanocrystal. By assuming the constant number of particles during the process, we have Equation (14).

$$
\Delta E_{g}^{-\frac{3}{2}} \propto t
$$

We presented a model to explain this phenomenon as shown in Figure 15. It is assumed that there is a local source of $\mathrm{H}^{+}$ions near the surface of nanoparticles.

There are two sources for $\mathrm{H}^{+}$ions: 1 ) the background $\mathrm{H}^{+}$ions that control the macroscopic $\mathrm{pH}$ value of the solution, and the local $\mathrm{H}^{+}$ions that are released by sticking to the TGA molecules as shown in Figure 16.

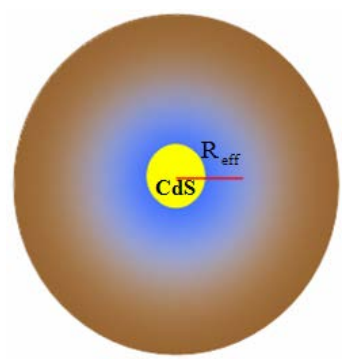

Figure 15. Presented model which dark areas show the background $\mathrm{H}^{+}$ions and light areas belong to local $\mathrm{H}^{+}$ions. 


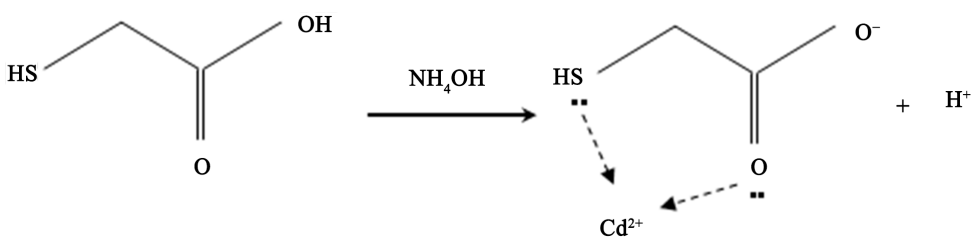

Figure 16. Mechanism of releasing $\mathrm{H}^{+}$ions via thiol molecules.

Released $\mathrm{H}^{+}$ions on the surface of nanoparticles are propagated toward the outside and will produce a gradient for concentration of $\mathrm{H}^{+}$ions. Since the density of local $\mathrm{H}^{+}$ions is decreased by increasing the particle's radius, we can assume a spherical region with an effective radius around the particle. When $\mathrm{pH}$ value is low, the density of $\mathrm{H}^{+}$ions is high and will quench the effect of local $\mathrm{H}^{+}$ ions.

By increasing $\mathrm{pH}$ value, the amount of background $\mathrm{H}^{+}$ions will be decreased and thus the effect of local ions would be dominant. In this case, we can define an effective radius that is decreased by $\mathrm{pH}$ increasing. The effective radius is the radius that has adequate $\mathrm{H}^{+}$ions for chemical reaction called threshold concentration. Also, while releasing an $\mathrm{H}^{+}$ion, there is most probable to be joined to $\mathrm{S}_{2} \mathrm{O}_{3}^{2-}$ or $\mathrm{OH}^{-}$ions. While increasing $\mathrm{pH}$ value, since with $50 \mathrm{mM}$ of $\mathrm{Na}_{2} \mathrm{~S}_{2} \mathrm{O}_{3}$, the amount of $\mathrm{S}_{2} \mathrm{O}_{3}^{2-}$ ions is much more than the amount of $\mathrm{OH}^{-}$ions, so the released $\mathrm{H}^{+}$ions will join to the $\mathrm{S}_{2} \mathrm{O}_{3}^{2-}$ ions and do not be neutralized by $\mathrm{H}^{+}$ ions.

\section{Conclusion}

This paper presents results supporting the synthesis of Mn-doped CdS semiconductor nanoparticles in the range between $3-7 \mathrm{~nm}$. The luminescence of the nanoparticles showed an increase by decreasing $\mathrm{Mn}$-to-Cd doping ratio. As a conclusion, the growth temperature of $96^{\circ} \mathrm{C}, \mathrm{Mn}$ :Cd doping ratio of 1:180 and $\mathrm{pH}=8$ were the optimized parameters for the synthesis of high luminescent Mn-doped CdS nanoparticles. Also a theoretical explanation based on quantum mechanics presented to justification the behavior of fabricated high luminescent Mn-doped CdS quantum dots.

\section{References}

[1] Koole, R., Groeneveld, E., Vanmaekelbergh, D., Meijerink, A. and de Mello Donegá, C. (2014) Size Effects on Semiconductor Nanoparticles. In: Nanoparticles. Workhorses of Nanoscience, Springer, Berlin, 13-51.

[2] Azizian-Kalandaragh, Y., Khodayari, A., Zeng, Z., Garoufalis, C.S., Baskoutas, S. and Gontard, L.C. (2013) Strong Quantum Confinement Effects in SnS Nanocrystals Produced by Ultrasound-Assisted Method. Journal of Nanoparticle Research, 15, 1-9. https://doi.org/10.1007/s11051-012-1388-1

[3] Lee, H., Issam, A., Belmahi, M., Assouar, M., Rinnert, H. and Alnot, M. (2009) Synthesis and Characterizations of Bare CdS Nanocrystals using Chemical Precipitation Method for Photoluminescence Application. Journal of Nanomaterials, 2009, Article ID: 914501. https://doi.org/10.1155/2009/914501 
[4] Xu, W., Zheng, C., Hua, H., Yang, Q., Chen, L., Xi, Y. and Hu, C. (2015) Synthesis and Photoelectrochemical Properties of CdWO4 and CdS/CdWO4 Nanostructures. Applied Surface Science, 327, 140-148. https://doi.org/10.1016/j.apsusc.2014.11.156

[5] Sankapal, B., Tirpude, A., Majumder, S. and Baviskar, P. (2015) 1-D Electron Path of 3-D Architecture Consisting of Dye Loaded CdS Nanowires: Dye Sensitized Solar Cell. Journal of Alloys and Compounds, 651, 399-404. https://doi.org/10.1016/j.jallcom.2015.08.116

[6] Li, X., Yang, Q., Hua, H., Chen, L., He, X., Hu, C. and Xi, Y. (2015) CdS/CdSe Core/Shell Nanowall Arrays for High Sensitive Photoelectrochemical Sensors. Journal of Alloys and Compounds, 630, 94-99. https://doi.org/10.1016/j.jallcom.2015.01.020

[7] Di, X., Kansal, S.K. and Deng, W. (2009) Preparation, Characterization and Photocatalytic Activity of Flowerlike Cadmium Sulfide Nanostructure. Separation and Purification Technology, 68, 61-64. https://doi.org/10.1016/j.seppur.2009.04.007

[8] Vempati, S. (2010) Comment on Semiconductor Nanocrystals: Structure, Properties, and Band Gap Engineering. Accounts of Chemical Research, 43, 190.

[9] Rohrmoser, S. (2010) Hybrid Optoelectronics with Colloidal Nanocrystals. PhD Thesis, University of Southampton, Southampton.

[10] Salavati-Niasari, M., Loghman-Estarki, M.R. and Davar, F. (2008) Controllable Synthesis of Nanocrystalline CdS with Different Morphologies by Hydrothermal Process in the Presence of Thioglycolic Acid. Chemical Engineering Journal, 145, 346-350. https://doi.org/10.1016/j.cej.2008.08.040

[11] Kosa, S.A. and Hegazy, E.Z. (2013) Extraction of Nanosized Cobalt Sulfide from Spent Hydrocracking Catalyst. Journal of Nanomaterials, 2013, Article ID: 471210. https://doi.org/10.1155/2013/471210

[12] Zou, B., Little, R., Wang, J. and El-Sayed, M. (1999) Effect of Different Capping Environments on the Optical Properties of CdS Nanoparticles in Reverse Micelles. International Journal of Quantum Chemistry, 72, 439-450.

[13] Kim, M.R., Kang, Y.-M. and Jang, D.-J. (2007) Synthesis and Characterization of Highly Luminescent CdS@ ZnS Core-Shell Nanorods. The Journal of Physical Chemistry C, 111, 18507-18511. https://doi.org/10.1021/jp075218n

[14] Bhattacharyya, S., Estrin, Y., Rich, D., Zitoun, D., Koltypin, Y. and Gedanken, A. (2010) Luminescent and Ferromagnetic CdS: $\mathrm{Mn}^{2+} / \mathrm{C}$ Core-Shell Nanocrystals. The Journal of Physical Chemistry C, 114, 22002-22011. https://doi.org/10.1021/jp107083f

[15] Marandi, M., Taghavinia, N. and Mahdavi, S. (2005) A Photochemical Method for Controlling the Size of CdS Nanoparticles. Nanotechnology, 16, 334. https://doi.org/10.1088/0957-4484/16/2/027

[16] Heiba, Z.K., Mohamed, M.B. and Imam, N. (2015) Biphasic Quantum Dots of Cubic and Hexagonal Mn Doped CdS; Necessity of Rietveld Analysis. Journal of Alloys and Compounds, 618, 280-286. https://doi.org/10.1016/j.jallcom.2014.08.106

[17] Cotter, D., Manning, R., Blow, K., Ellis, A., Kelly, A., Nesset, D., Phillips, I., Poustie, A. and Rogers, D. (1999) Nonlinear Optics for High-Speed Digital Information Processing. Science, 286, 1523-1528. https://doi.org/10.1126/science.286.5444.1523

[18] Thilak, T., Ahamed, M.B. and Vinitha, G. (2013) Third Order Nonlinear Optical Properties of Potassium Dichromate Single Crystals by Z-Scan Technique. OPtik-International Journal for Light and Electron Optics, 124, 4716-4720. https://doi.org/10.1016/j.ijleo.2013.01.111 
[19] Zeng, H., Han, J., Qian, D. and Gu, Y. (2014) Third-Order Nonlinear Optical Properties of Multiwalled Carbon Nanotubes Modified by CdS Nanoparticles. $O p$ tik-International Journal for Light and Electron Optics, 125, 6558-6561. https://doi.org/10.1016/j.ijleo.2014.06.166

[20] Ganesan, P. and Senthilkumar, L. (2015) The Influence of Interfaces and Intra-Band Transitions on the Band Gap of CdS/HgS and GaN/X (X = InN, $\operatorname{In}_{0.33} \mathrm{Ga}_{0.67} \mathrm{~N}$ ) Core/Shell/Shell Quantum Dot Quantum Well-A Theoretical Study. Physica E: Low-Dimensional Systems and Nanostructures, 74, 204-212. https://doi.org/10.1016/j.physe.2015.07.002

[21] Mary, K.A., Unnikrishnan, N. and Philip, R. (2015) Defects Related Emission and Nanosecond Optical Power Limiting in CuS Quantum Dots. Physica E: Low-Dimensional Systems and Nanostructures, 74, 151-155. https://doi.org/10.1016/j.physe.2015.06.031

[22] Wang, W., Yu, L., Yang, H., Hong, K., Qiao, Z. and Wang, H. (2015) Growth Mechanism of $\mathrm{ZnO}$ Nanorod/ $\mathrm{Fe}_{3} \mathrm{O}_{4}$ Nanoparticle Composites and Their Photocatalytic Properties. Physica E: Low-Dimensional Systems and Nanostructures, 74, 71-73. https://doi.org/10.1016/j.physe.2015.06.024

[23] Qutub, N., Pirzada, B.M., Umar, K., Mehraj, O., Muneer, M. and Sabir, S. (2015) Synthesis, Characterization and Visible-Light Driven Photocatalysis by Differently Structured CdS/ZnS Sandwich and Core-Shell Nanocomposites. Physica E: Low-Dimensional Systems and Nanostructures, 74, 74-86. https://doi.org/10.1016/j.physe.2015.06.023

[24] Wang, J., Gao, Y., Ng, M.-Y. and Chang, Y.-C. (2015) Radial Vibration of Ultra-Small Nanoparticles with Surface Effects. Journal of Physics and Chemistry of Solids, 85, 287-292. https://doi.org/10.1016/j.jpcs.2015.06.005

[25] Smirnova, D.A., Shadrivov, I.V., Miroshnichenko, A.E., Smirnov, A.I. and Kivshar, Y.S. (2014) Second-Harmonic Generation by a Graphene Nanoparticle. Physical Review B, 90, Article ID: 035412. https://doi.org/10.1103/PhysRevB.90.035412

[26] Yao, Y., Wei, Y. and Chen, S. (2015) Size Effect of the Surface Energy Density of Nanoparticles. Surface Science, 636, 19-24. https://doi.org/10.1016/j.susc.2015.01.016

[27] Scherbak, S., Shustova, O., Zhurikhina, V. and Lipovskii, A. (2014) Electric Properties of Hemispherical Metal Nanoparticles: Influence of the Dielectric Substrate. Journal of Physics. Conference Series, 541, Article ID: 012077. https://doi.org/10.1088/1742-6596/541/1/012077

[28] Matyushov, D.V. (2014) Electrophoretic Mobility without Charge Driven by Polarisation of the Nanoparticle-Water Interface. Molecular Physics, 112, 2029-2039. https://doi.org/10.1080/00268976.2014.882521

[29] Demetriadou, A. and Kornyshev, A.A. (2015) Principles of Nanoparticle Imaging using Surface Plasmons. New Journal of Physics, 17, Article ID: 013041. https://doi.org/10.1088/1367-2630/17/1/013041

[30] Gan, L. (2013) Device, System, and Method of Frequency Generation using an Atomic Resonator. Patent US9685909 B2.

[31] Wageh, S., Maize, M., Han, S., Al-Ghamdi, A.A. and Fang, X. (2014) Effect of Solvent and Environmental Conditions on the Structural and Optical Properties of CdS Nanoparticles. RSC Advances, 4, 24110-24118. https://doi.org/10.1039/C4RA03139C

[32] Bansal, P., Jaggi, N. and Rohilla, S. (2012) Green Synthesis of CdS Nanoparticles and Effect of Capping Agent Concentration on Crystallite Size. Research Journal of 
Chemical Sciences, 2231, 606X.

[33] Guo, L., Jing, D., Liu, M., Chen, Y., Shen, S., Shi, J. and Zhang, K. (2014) Functionalized Nanostructures for Enhanced Photocatalytic Performance under Solar Light. Beilstein Journal of Nanotechnology, 5, 994-1004.

https://doi.org/10.3762/bjnano.5.113

[34] Talapin, D.V., Nelson, J.H., Shevchenko, E.V., Aloni, S., Sadtler, B. and Alivisatos, A.P. (2007) Seeded Growth of Highly Luminescent CdSe/CdS Nanoheterostructures with Rod and Tetrapod Morphologies. Nano Letters, 7, 2951-2959. https://doi.org/10.1021/nl072003g

[35] Zhang, Y.-P., Liu, W., Liu, B.-D. and Wang, R.-M. (2014) Morphology-Structure Diversity of ZnS Nanostructures and Their Optical Properties. Rare Metals, 33, 1-15. https://doi.org/10.1007/s12598-013-0217-8

[36] Nabi, A. (2016) The Electronic and the Magnetic Properties of Mn Doped Wurtzite CdS: First-Principles Calculations. Computational Materials Science, 112, 210-218. https://doi.org/10.1016/j.commatsci.2015.10.039

[37] Borse, P., Srinivas, D., Shinde, R., Date, S., Vogel, W. and Kulkarni, S. (1999) Effect of $\mathrm{Mn}^{2+}$ Concentration in $\mathrm{ZnS}$ Nanoparticles on Photoluminescence and Electron-Spin-Resonance Spectra. Physical Review B, 60, 8659 . https://doi.org/10.1103/PhysRevB.60.8659

[38] Dantas, N.O., Fernandes, G.L., Baffa, O., Gomez, J.A. and Almeida Silva, A.C. (2015) Controlling Density of Manganese Ions and Cadmium Vacancies into $\mathrm{Cd}_{1-\mathrm{x}} \mathrm{Mn}_{\mathrm{x}} \mathrm{Te}$ Ultrasmall Quantum Dots in Glass Matrix: X-Concentration and Thermal Annealing. The Journal of Physical Chemistry C, 119, 17416-17420.

[39] Liu, Y., Xiao, N., Gong, N., Wang, H., Shi, X., Gu, W. and Ye, L. (2014) One-Step Microwave-Assisted Polyol Synthesis of Green Luminescent Carbon Dots as Optical Nanoprobes. Carbon, 68, 258-264. https://doi.org/10.1016/j.carbon.2013.10.086

[40] Oluwafemi, O.S., Daramola, O.A. and Ncapayi, V. (2014) A Facile Green Synthesis of Type II Water Soluble CdTe/CdS Core Shell Nanoparticles. Materials Letters, 133, 9-13. https://doi.org/10.1016/j.matlet.2014.06.152 